\title{
Speed and Accuracy Indicators of Test Performance Under Different Instructional Conditions: \\ Intelligence Correlates
}

Running Head: Speed and Accuracy

Submitted to: Intelligence

Original Submission: December 13, 2015

Revised Submission: January 31, 2016 


\section{ABSTRACT}

Performance on speeded ability tests, in contrast to power tests, reflects an individual's ability to get answers correct and to do so rapidly. However, with speeded tests, overall performance scores represent some unknown combination of the individual's strategy toward greater speed or higher accuracy. Scoring methods, such as penalties for wrong answers, are often imposed to either encourage examinees to adopt a specific speed-accuracy tradeoff strategy, or to attempt to derive performance scores that 'factor out' such strategic differences. In the current study, baseline assessments of four perceptual speed and psychomotor ability tests were administered, along with three different instructional conditions (accuracy-emphasis, speed-emphasis, and balanced accuracy and speed). A general ability composite was derived from a battery of intellectual ability tests. Changes in speed-accuracy tradeoff emphasis resulted in a consistent pattern of changes in the $g$ correlates of latency/completion time performance indicators and number of errors. Increasing emphasis on accuracy resulted in increasing $g$ correlates with latency/completion time, and decreases in $g$ correlates with error rates. Implications for construct validity of ability tests and for further consideration of the conditions of testing are presented.

\section{Background}

From the early years of modern testing, there has been a robust debate about the importance of speed and level of performance on ability tests, as they relate to the overall construct of intelligence. E. L. Thorndike et al. (1926), for example, noted that "Other things being equal, the more quickly a person produces the correct response, the greater is his [sic] intelligence” (p. 24, italics in original). That is, Thorndike considered speed to represent an 
intellectual advantage when individuals were equivalent on "level." In practice, however, it is difficult or impossible to have examinees perform at an equal level, but differ only on speed.

Early in testing research and applications, the main concern of investigators was whether or not increasing testing time to reduce the speed-demands of tests resulted in differential effects on the rank-ordering of individuals on test performance (e.g., Beck, 1933; Tinker, 1931 for early reviews). The results of such investigations were generally mixed—some tests with different speed constraints yielded correlations close to the individual test reliabilities, while others did not. Thorndike, in fact, recognized that individual differences in time management could result in higher or lower test scores, but he concluded that such differences were not of substantial importance (E. L. Thorndike et al., 1926).

Later investigators were better aware of the potential measurement issues associated with the fact that individuals could trade-off accuracy for speed on some tests. That is, there is a fundamental dependence between how rapidly one completes test items and how accurate (correct) the answers are. Most researchers attempted to grapple with this issue by trying to equalize performance of individuals who adopted different speed-accuracy tradeoff strategies. For example, discussions have been accorded to determining the 'best' (from reliability and validity perspectives) methods for computing the total score for multiple-choice tests. Numerous alternate formulas have been proposed. Such formulas typically either provide no credit for incorrect responses or impose a penalty in an attempt to 'correct for guessing' on other items for which one can reasonably infer the examinee did not actually know the correct answer, but managed to guess correctly (e.g., see Lord, 1975, for a review; see also Diamond \& Evans, 1973). 
When test items vary considerably in terms of difficulty, however, there are multiple causes of wrong answers to test items. The examinee may be running out of time on a timed test with multiple items uncompleted, so the examinee may randomly guess the answers on the remaining test items. If that is the case, then a correction for guessing that takes account of such random responding might subtract a fraction of a point for each incorrect response (where the fraction is equal to one divided by the number of item response options). If the test developer wishes to discourage guessing entirely, then a subtraction for wrong answers that is larger than one over the number of item response options would presumably yield an appropriate penalty. Yet, in operational situations, examinees often have partial knowledge about the correctness or incorrectness of response options. In such cases, when penalties for wrong answers are known, the examinee might evaluate how many wrong options can be eliminated from consideration, and then determine whether the chances of getting the item wrong are lower than the penalty for guessing — leading to a decision of whether a partially-informed guess for that item is worth the risk.

For constructed response test items (e.g., sentence completion tests), however, the chances for successful guessing are generally unknown, but often are believed to be very low. For these kinds of tests, developers typically see little need to correct scores for guessing, and simply sum the number of correct answers to obtain a final test score. Similarly, for power tests (which are traditionally untimed), investigators have largely ignored errors, and focused only on the number of correct answers. However, it is not clear whether salient individual differences exist in a propensity to take short-cuts by guessing about responses, when the examinee does not recognize the correct answer. 
For speeded tests, in which items are sometimes relatively homogeneous in difficulty, such as tests of basic math (subtraction and addition), perceptual speed, and psychomotor abilities - the assumption that examinee 'knowledge' will mainly determine item response accuracy is generally untenable. This is primarily because the expectation is that with unlimited time and a modicum of motivation (e.g., see Thurstone, 1937), test performance would be errorfree. For such tests, the underlying perspective is that there is a speed-accuracy tradeoff function for each individual, depending on his/her ability level (e.g., see Lohman, 1989a). When intelligence investigators first adopted the information processing perspective for ability testing in particular, there was an active controversy about whether speed of responding to simple items is a salient indicator of intellectual ability (e.g., see Jensen, 1998, 2006; Vernon, Nador, \& Kantor, 1985; though see Sternberg, 1986 for an opposing viewpoint).

The problem of speed-accuracy tradeoff (Wickelgren, 1977) is that the function that describes the relationship is generally found to be decidedly non-linear. Accuracy increases rapidly with decreasing speed initially, but as accuracy reaches asymptotic levels, latency tends toward infinity (i.e., diminishing returns on accuracy with increasing latency). Performance on most group ability tests is the total number of items correctly answered (or total number of items answered correctly minus some fraction of the total number of items marked incorrect). However, for speeded tests, the total number of items answered correctly is the result of an unknown combination of the examinee's knowledge and his or her ability or motivation to answer items quickly. Compared to lower ability examinees, higher ability examinees are presumed to respond more accurately at a given response speed, or with shorter response latencies at a given level of accuracy, in line with E. L. Thorndike and colleagues' (1926) 
suggestion. However, the precise shape of each individual examinee's speed-accuracy tradeoff function is generally unknown. This makes it impossible to determine each individual's location on his or her own speed-accuracy tradeoff function, based only on a single latency and error score. Therefore, in most testing situations, it is generally impossible to determine which of two individuals has higher ability, unless one individual is both faster and more accurate than the other individual. Furthermore, because the shape of the speed-accuracy tradeoff function is unknown and likely nonlinear under normal testing conditions, it is not feasible to derive an accurate 'correction for guessing' for speeded tests that is as straightforward as that used for traditional multiple-choice tests. Some approaches to determining speed-accuracy tradeoff functions have been tried, such as imposing different response deadlines or varying the amount of time an item is displayed, most notable is the work by Lohman $(1986,1989 \mathrm{a}, 1989 \mathrm{~b})$ on spatial visualization tests. Other approaches have examined the reliability/consistency of tests, and the reactivity of individuals, when confronted with tests that are administered with explicit manipulation of payoffs for right and wrong answers (e.g., Quereshi, 1960). Nonetheless, many tests retain a simple fractional penalty for guessing, even when the items are homogeneous in difficulty levels, such as tests of perceptual speed ability (e.g. see Ekstrom, French, Harman, \& Dermen, 1974).

Numerous studies have attempted to address the implications of speed-accuracy tradeoffs for ability testing, whether in terms of: (a) providing explicit instructions regarding accuracy and errors; (b) deriving test scores that attempt to equate scores of individuals who appear to differ on an emphasis of speed or accuracy; (c) exploring derivations of individual speed-accuracy tradeoff functions; or (d) manipulating speed-accuracy tradeoff by changing payoff matrices for 
correct answers and errors. However, little is known about how changes in instructional emphasis for speed vs. accuracy affect the construct validity of tests, especially in terms of the $g$ loading of performance measures. This question is important because tests that were designed to be administered under relatively liberal time limits are sometimes administered under speeded conditions in research settings (such as Raven's Progressive Matrices in some working memory research; for a review and meta-analysis, see Ackerman, Beier, \& Boyle, 2002, 2005). This question is difficult to address using power tests containing items of varying levels of difficulty, because speed/accuracy tradeoff will be confounded with item difficulty, making the results difficult to interpret. However, tests composed of items of uniform difficulty allow more straightforward manipulation of the speed-accuracy tradeoff and interpretation of results.. The current study focused on a small set of such tests and a set of reference ability measures, in order to investigate changes in $g$-loadings in response to speed-accuracy instructional manipulations.

\section{Current Study}

In the current study, the main question of interest was whether different instructional emphases on speed or accuracy influence the $g$-loading of various tests, and whether these instructional effects were reflected in separate latency and accuracy scores for the tests. To explore this question, we selected a set of perceptual speed and psychomotor tests, mainly because they have two advantages over other kinds of tests. One principal advantage of these tests is that they typically involve items of roughly equal difficulty, which means that the underlying speed/accuracy function for each item is essentially equivalent. The second advantage is that, in contrast to traditional fluid or crystallized intelligence tests, the test items can be answered correctly without error in untimed administrations (e.g., see Ackerman, 1988). 
Thus, errors on such tests are entirely a result of the examinees' internal rules or experimenterimposed instructions for speed-accuracy emphases. However, one disadvantage to such tests that must be noted is that there are typically significantly larger 'practice' effects, compared to crystallized or fluid intellectual ability tests. The presence of practice effects means that one should either provide initial practice (to improve the stability of test performance) on the tests prior to instructional manipulations, or counterbalance the order of instructional manipulations (or both), to avoid carry-over confounds from order effects.

Tests that make demands on perceptual speed and psychomotor abilities—especially those that include more complex processing than simple reaction time or cancellation testshave historically been included in omnibus intelligence tests (e.g., the digit-symbol subtest in the Wechsler Scales). Complex perceptual speed and psychomotor tests often correlate substantially with other measures of general intelligence, especially those that are speeded to some degree (e.g., see Ackerman \& Cianciolo, 1999; Alderton, Wolfe, \& Larson, 1997; Allison, 1960; Melton, 1947). These tests also often have significant incremental predictive validity (beyond non-speeded $g$ measures) for occupational performance, such as in aviation and dentistry domains (Ackerman \& Kanfer, 1993). Nonetheless, modern intelligence test batteries have removed these tests - not because of a lack of construct or criterion-related validity, but because pre-computer versions of such tests often required specialized apparatus for administration (Fleishman, 1953).

The general layout for the study was as follows. First, a series of paper and pencil tests assessing verbal, numerical, and spatial abilities (two of each) were administered, in order to provide a robust assessment of general intellectual ability. Next, two perceptual speed tests 
(Summing to 10 and Scattered X's) and two psychomotor tests (Eight-item Serial Reaction Time [SRT] and Mirror Tracing) were administered for baseline assessments, with generic instructions to focus on speed while maintaining a low level of errors, in order to provide examinees with initial familiarization and practice. In a follow-up session, the perceptual speed and psychomotor tests were administered under three different instructional conditions: Accuracy (in which examinees were instructed to devote $90 \%$ of their effort to maintaining accurate performance); Balanced (in which examinees were instructed to give equal priority to speed and accuracy); and Speed (in which examinees were instructed to give $90 \%$ of their effort to performing as quickly as possible), with a Latin Square ordering of the three instructional conditions. The Latin Square ordering results in a counterbalanced design, so that equal portions of the participants received the accuracy conditions first, the balanced conditions first, and the speed conditions first. As a result of this counterbalancing, any carry-over effects from the different instructional conditions are relegated to the error term.

\subsection{Hypotheses}

Two overarching results need to be established prior to evaluating the relationship between general intellectual ability and test performance under different speed-accuracy emphasis instructional conditions. First, the set of ability measures administered to represent $g$ must load substantially on a single general factor, so that a composite $g$ measure can be derived. Second, performance on the criterion tests must show overall changes in speed and accuracy that match the instructional emphasis (i.e., item response latencies must decrease, and error rates must increase, from accuracy emphasis instructions, to balanced emphasis instructions, to speed emphasis instructions). Rather than being experimental hypotheses, these are essentially checks 
on the overall study rationale. In addition, it is important that, at least in the baseline test performance measures, performance on the criterion tests be significantly correlated with $g$, or it would not be clear that the tests are tapping abilities that are associated with general intellectual ability.

If the above conditions are met, the overarching research questions pertaining to the differential relationships between $g$ and speed (latency) and accuracy (error) rates under the different instructional conditions can be tested. The general conjecture is that when speed or accuracy is emphasized, individual differences in performance will be most highly associated with $g$ on the variables that are given the greatest emphasis. That is, when individuals are encouraged to work to maximize speed, then latency/completion times will be most highly related to $g$, and conversely, when individuals are encouraged to work to maximize accuracy, then the number of errors made during the test will be most highly related to $g$. (Because we did not emphasize either accuracy to the complete exclusion of speed, or speed to the complete exclusion of accuracy, we did not expect participants to entirely ignore these other aspects. For example, in a 'complete' accuracy-emphasis condition, examinees would be expected to have zero errors, given that the items, as noted earlier, are easy enough to allow for perfect performance, given enough time.)

In particular, we hypothesized that under accuracy emphasis conditions, error rates would be most highly related to $g$, and speed will be least related to $g$. Under speed emphasis conditions, latency would be most highly related to $g$, and accuracy would be least related to $g$. Under balanced conditions, performance measures (both latency and errors) would be in between those of the accuracy and speed emphasis condition relations with $g$. Thus: 
H1: Across the four tests, there should be an increasing correlation between latency/completion time and $g$, from accuracy-emphasis to balanced-emphasis to speedemphasis.

H2: Across the four tests, there should be a decreasing correlation between error rate and $g$, from accuracy-emphasis to balanced-emphasis to speed-emphasis.

Note that, because longer latencies and higher error rates both represent poorer performance, the overall correlations between these measures and $g$ are expected to be negative. That is, shorter latencies (faster performance) and lower error rates are expected to be positively associated with

$g$.

\section{Method}

\subsection{Participants}

One hundred participants were recruited via in-class announcements and flyers posted on college campuses and public places in Atlanta, Georgia, USA. Inclusion criteria were: (a) 18-25 years old; (b) fluent in English; (c) normal or corrected-to-normal hearing, vision, and motor coordination; and (d) high-school graduate. Although only a high-school diploma was required, all but four participants were currently enrolled in a post-secondary institution. Data for two participants were eliminated for failure to follow instructions. The final sample included 98 participants (43 women, 55 men). Participants were compensated $\$ 100$ for completing the study, which included two sessions of four hours each. ${ }^{1}$

\subsection{Ability Test Battery}

${ }^{1}$ The study also included a variety of other measures unrelated to the issues of speedaccuracy tradeoff, which are not discussed here 


\subsubsection{Verbal ability}

Verbal ability was assessed using two tests. In the Extended Range Vocabulary test (Ekstrom et al., 1976), respondents select the response option that has the same or nearly the same meaning as a target word. The test was presented in two parts of 24 items each, and participants had 6 minutes to complete each part. Score was the number of correct items. Cronbach's alpha ranged from $\alpha=.78$ to .92 in a study by Ackerman, Bowen, Beier, and Kanfer (2001). In the Multidimensional Aptitude Battery: Similarities test (Jackson, 1984), participants must select the response option that best describes how two words are alike. Participants had 7 minutes to complete the 34 -item test. Score was the number of items correct. Cronbach's alpha ranged from $\alpha=.64$ to $\alpha=.84$ in Ackerman et al. (2001).

\subsubsection{Numerical ability.}

Numerical ability was assessed with two tests. The 40-item Math Approximation test (Ackerman \& Beier, 2007) was administered in two parts of 4.5 minutes each. The examinee is asked to compute an approximate solution to each item, and choose from five answer choices. Score was the total number of items correct. Cronbach's alpha ranged from $\alpha=.84$ to $\alpha=.91$ (Ackerman \& Beier, 2007). The Arithmetic test (from the Multi-Ability Test; Cureton \& Cureton, 1955) consists of math problems that require various arithmetic operations, such as adding mixed numbers and reducing to the lowest terms. The 20 -item test was administered in two parts of 4 minutes each. Score was the total number of items correct. Cronbach's alpha values ranged from $\alpha=.72$ to $\alpha=.79$ in Ackerman and Beier (2007).

\subsubsection{Spatial ability}

The Paper Folding test and the Spatial Analogy test were used to assess spatial ability. 
The Paper Folding test (Ackerman \& Kanfer, 1993) requires examinees to visualize the result when a piece of paper is folded, punched with a hole, and then unfolded. Four response options are presented. The 24-item test is administered in two 6-minute parts. Score was the total number of items correct. Cronbach's alpha ranged from $\alpha=.83$ to $\alpha=.86$ in Ackerman and Beier (2007). The Spatial Analogy test (Ackerman \& Kanfer, 1993) is structurally similar to verbal analogy tests, but items consist of geometric shapes. Examinees select from four answer choices to complete the analogy. Participants were given 9 minutes to complete this 30 -item test. Score was the total number of items correct. Cronbach's alpha ranged from $\alpha=.79$ to $\alpha=.88$ in Ackerman and Beier (2007).

\subsection{Perceptual Speed and Psychomotor Assessments}

The perceptual speed and psychomotor tests were administered on computers with 3M/Microtouch touch-sensitive monitors equipped with a TouchPen stylus. See Figure 1 for screen captures of the Perceptual Speed and Psychomotor tests.

\subsubsection{Perceptual Speed. Two perceptual speed tests were administered on the computer: the} Scattered X's test and the Summing to 10 test.

Scattered X's test. In this test, participants identified X's (by tapping their location on the touchscreen) in a field of irregularly spaced letters (Ackerman \& Beier, 2007; adapted from a design described in Thurstone \& Thurstone, 1941). Participants advanced to the next screen by tapping a button when they believed that they had located all X's on the current screen. In previous studies, internal consistency reliability for this test ranged from $r_{x x}=.95$ to .96 , and testretest reliability was $r_{a b}=.80$. In standard test conditions (i.e., not emphasizing speed or accuracy), marked performance correlates with $g$ are seen mainly with latency measures ( $r=-.50$ 
[latency], $r=-.15$ [errors]; from a study reported by Ackerman \& Beier, 2007).

Summing to 10 test. In this test, an array of single-digit number pairs is presented, and the examinee must identify those pairs whose component numbers sum to 10 . In previous studies, internal consistency reliability ranged from $r_{x x}=.92$ to .94 , and test-retest reliability ranged from $r_{a b}=.76$ to .82 . Similar to the Scattered X's test, in standard test conditions, marked performance correlates for the Summing to 10 test with $g$ are seen mainly with latency measures $(r=-.38$ [latency], $r=-.07$ [errors]; from a study reported by Ackerman \& Beier, 2007; see also Ackerman \& Cianciolo, 2000).

The Scattered X's and Summing to 10 tests were both presented in three 5-minute blocks for the baseline assessments and for each of the three speed-accuracy emphasis conditions.

\subsubsection{Psychomotor Ability}

Psychomotor ability was assessed with two tests: an Eight-item Serial Reaction Time (SRT) test and a Mirror Tracing test.

Eight-item SRT test. For the SRT (Ackerman \& Cianciolo, 2000; see also Ackerman \& Beier, 2007), participants held the TouchPen to a home key in the center of the screen, where the target boxes are all shown in a solid color. After a random delay of 400 to $800 \mathrm{~ms}$., the target boxes are replaced with randomly ordered numbers (1-8) -- see Figure 1. Participants were instructed to tap each target block in numerical order. Five blocks of 30 trials were presented during the baseline assessment and for each of the speed-accuracy emphasis conditions. Performance was measured as mean total completion time in milliseconds, and was presented after each trial. Only correct trials were included in this computation. Internal consistency reliability was $r_{x x}=.94$ (Ackerman \& Beier, 2007). Similar to the perceptual speed tests, in 
standard test conditions, marked performance correlates for the SRT test with $g$ are seen mainly with latency measures $(r=-.44$ [latency], $r=.07$ [errors]; from a study reported by Ackerman \& Beier, 2007)

Mirror Tracing test. In this test, a figure (e.g., a six-pointed star) was presented on the right side of the computer screen, and participants traced the shape on the left side of the screen, which was blank except for a home key. As the examinee traced the shape, the traced line appeared on the shape on the right side of the screen. However, all movements were mirrored left-to-right and top-to-bottom, such that an upward movement with the TouchPen on the left side of the screen resulted in a downward movement of the traced line on the right side of the screen. Twenty-seven trials were presented during baseline assessment. Performance was assessed using mean total completion time and number of errors (each time the tracing line went outside the boundary of the figure was counted as an error). From data collected in a previous study, internal consistency reliability was $r_{x x}=.94$. In contrast to the other tests presented above, however, correlations between Mirror Tracing performance and $g$ were notable for both completion time and number of errors $(r=-.44 * *$ [completion time] and $r=-.51$ [errors]; see Ackerman \& Cianciolo, 2000).

The number of trials for both the SRT and Mirror Tracing tests was selected in order to provide approximately 15 minutes of performance time for baseline and each speed-accuracy emphasis condition.

\subsection{Speed-accuracy Instructional Conditions}

For the four tests, equivalent instructions were provided regarding emphasis on accuracy, balanced, and speed conditions. For example, for the Eight-item SRT test, the following 
instructions were provided on the computer display and simultaneously read to the examinee over headphones:

3.4.1 Accuracy emphasis: "For the upcoming serial reaction time trials, we want you to do the task as ACCURATELY as you can. This means that you should not worry about how fast you tap the boxes. Just try to make sure you tap the 8 boxes in order, with as few errors as you can. For example, if you complete a block of trials with several errors, you are probably going too fast. If that happens, try to slow down on the next trials so that you can make fewer errors. Think of doing the task with $90 \%$ of your effort devoted to accuracy, but only $10 \%$ percent of your effort devoted toward speed."

3.4.2. Balanced emphasis: "For the upcoming serial reaction time trials, we want you to do the task with BALANCED emphasis on speed and accuracy. This means that you should focus equally on the speed of tapping the 8 boxes in order, and on not making errors. Think of doing the task with $50 \%$ of your effort devoted toward speed, and $50 \%$ percent of your effort devoted toward keeping the number of errors low."

3.4.3. Speed emphasis: "For the upcoming serial reaction time trials, we want you to do the task as FAST as you can. This means that you should not worry too much about how many errors you make. Just try to get all 8 boxes tapped in order, as fast as you can. For example, if you get through an entire block of 25 trials with no errors, you are probably not going as fast as you can. If that happens, try to go even faster on the next block of trials. Think of doing the task with $90 \%$ of your effort devoted to speed, but only $10 \%$ percent of your effort devoted toward keeping the number of errors low."

Reminders of the instructional condition were also given after each block of trials for 
each test/condition.

\section{Results}

The results of the study are described in three sections. First, evaluation of the reference tests is provided, along with a derivation of a $g$ composite. Next, performance on the perceptual speed and psychomotor tests is examined, to determine whether changes in speed-accuracy emphasis instructions had the expected effects on latency and error measures. In the third section, we examine the respective correlations between the $g$ composite and latency and error measures for each test, both at baseline and in the three instructional conditions, to evaluate the two hypotheses.

\subsection{Estimating $g$}

Descriptive statistics for the six ability reference tests are provided in Table 1, including means, sds, internal consistency reliabilities, and intercorrelations. Although three factors could reasonably be extracted from the correlation matrix, our main concern was with a single general factor. With principal axis factoring, a single factor was extracted, with the loadings also indicated in Table 1. Following suggestions from R. L. Thorndike (1986) and Cohen (1990), we converted the individual test scores to $z$-scores, and created an unweighted $z$-score composite for the $g$ estimate. (With component test scores as 'items,' the coefficient alpha for the $g$ composite was $\alpha=.82$, indicating a reasonably high consistency for the reference ability tests. $)^{2}$

\subsection{General Patterns of Speed-Accuracy Tradeoff}

Descriptive statistics for the four criterion tests are provided in Table 2, for both baseline

${ }^{2}$ The $g$ composite made up of verbal, math, and spatial abilities had nearly identical rankordering of participants, when compared to a composite composed of only verbal and math abilities $(r=.95)$. 
and speed-accuracy emphasis instruction conditions. For each test, performance has been averaged across multiple blocks of trials to obtain approximately 15 minutes of time-on-test for each test and condition (i.e., three 5-minute test blocks for both the Scattered X's and Summing to 10 tests, 5 blocks of 30 trials for the Eight-item SRT test, 3 blocks of 10 trials for the Mirror Tracing test). Means and sds for each test are presented. For the Scattered X's and Summing to 10 tests, latency measures were computed by dividing 300 (the number of seconds in each 5minute test part) by the average number of items correctly identified in each 5-minute block. This provides an average latency score that reflects the number of seconds needed to identify each correct item, and is comparable to the other latency measures (average time in seconds to complete one item). Latency measures are provided in the upper section of the table, and error rates are provided in the lower section of the table.

In addition, repeated measures ANOVAs were conducted to determine whether there were significant changes in latency and error measures from accuracy-emphasis to balancedemphasis to speed-emphasis. The main effect of instructional condition is presented for each criterion measure in Table 2, along with an estimate of effect size (partial eta squared). As shown in the table, instructional condition had a significant and substantial effect on the latency and error rates, reflecting decreases in latency and increases in error rates, from accuracyemphasis to balanced-emphasis to speed-emphasis. Based on these results, we conclude that the instructional manipulation had the effects of altering overall latency and error rates in the direction that was expected.

Also, keeping in mind that the Latin Square design of condition administrations likely resulted in some deviations in performance attributable to practice effects, we counted the 
number of participants whose latency/completion time during the speed-emphasis condition was faster overall than their performance in the accuracy-emphasis conditions, across the four tests. Nearly half (46) of the participants showed this pattern in all four tests, another third of the participants (33) showed this pattern for three of the four tests, 12 showed it in two of the tests, five for one test, and two participants had this pattern for none of the tests. For error rates, similar results were obtained. That is, roughly half (51) of the participants had higher error rates in the speed conditions across all four tests, one third of the participants (34) had higher error rates in three tests, 12 showed this pattern in two of the tests, and one participant had this pattern in only one of the tests. These results are generally suggestive of the notion that most of the participants were able to adjust their personal speed-accuracy tradeoff strategies in response to the task emphasis instructions.

\subsection{Correlations with $g$}

Correlations between performance on each of the perceptual speed and psychomotor tests and the $g$ composite are shown in Table $\mathbf{3}$ and illustrated graphically in Figure 2, for baseline assessments and under each of the speed-accuracy emphasis conditions. At baseline, latency measures for all four tests were significantly correlated with the $g$ composite. For errors, only the Mirror Tracing test was substantially $(r=-.50)$ correlated with $g$, though there was also a significant correlation between $g$ and errors on the Scattered X's test $(r=-.28)$ at baseline. The other two tests had negligible error rate correlations with $g$. One inference from these correlations is that for two of the tests (i.e., Mirror Tracing and Scattered X's), higher ability examinees performed both faster and more accurately than lower ability examinees. On the other two tests, higher-ability examinees were faster, but no more or less accurate, on average, 
compared to lower-ability examinees. These results are consistent with the notions that either the examinees adopted a similar emphasis on speed and accuracy, or there was not a marked pattern of differences in individual tendencies toward speed or accuracy associated with differences in general intellectual ability.

When examining the respective latency correlations with $g$ under the different speedaccuracy emphasis conditions, it is clear that for all four tests, the smallest correlations with $g$ were found under accuracy-emphasis conditions, and the largest correlations were found under the speed-emphasis conditions (consistent with H1). In contrast, the opposite pattern was found for error measures. That is, the largest correlations with $g$ were found for the accuracy-emphasis conditions, and the smallest correlations with $g$ were found for the speed conditions (consistent with H2). In order to assess the significance of differences between the correlations with $g$, we focused on the most extreme instructional condition differences (i.e., accuracy-emphasis and speed-emphasis). In assessing these differences in correlations, it is necessary to take account of the correlations between performance under different conditions for the same tasks, as higher correlations indicate higher power to detect differences. In addition, these correlations illustrate the consistency in rank-ordering of individuals across the different instructional conditions within the same tasks. For latency measures, correlations between the accuracy-emphasis and speed-emphasis conditions were positive and significant, but indicated a large range (Scattered $\mathrm{Xs}, r=.44$, Summing to $10, r=.74$, SRT, $r=.64$, and Mirror Tracing, $r=.35$, all $p<.01$ ), suggesting that for at least the Scattered Xs and Mirror Tracing tests, there was a substantial reordering of individuals in test performance across the instructional conditions. For the error measures, correlations between instructional conditions were much more modest (Scattered Xs, $r$ 
$=.20$, Summing to $10, r=.16$, SRT, $r=.26$, and Mirror Tracing, $r=.33$; only SRT and Mirror Tracing were significantly different from zero, $p<.05)$.

Differences between correlations with $g$ under accuracy-emphasis and speed-emphasis conditions were assessed using Williams's $\mathrm{T}_{2}$ test for differences between dependent correlations (Steiger, 1980). As shown in Table 3, results of this test indicated significant differences in correlations with latency for the two psychomotor tasks (SRT: $T_{2}=-4.11, p<.01$; Mirror Tracing: $\left.T_{2}=-3.34, p<.01\right)$. As expected, the correlations between latency and $g$ were stronger in the speed-emphasis condition than in the accuracy-emphasis condition. For the error measures, all four tests showed significant differences in the strength of correlations with $g$ such that correlations were stronger under accuracy-emphasis conditions than under speed-emphasis conditions. (See Table 3.)

\section{Discussion}

At baseline administration of the two perceptual speed and psychomotor tests, latency measures were significantly, and in the case of three of the tests, substantially related to the general ability $(g)$ composite. Error rates on the same tests, however, had a different patternonly two of the tests had error rates significantly correlated with $g$ at baseline, and only the Mirror Tracing test showed a substantial correlation between error rates and $g$. One take-away from such results is that performance (especially latency) on these tests is consistently associated with general intellectual ability. Historically, estimates of the $g$-loadings for perceptual speed and psychomotor tests have been modest, but these may be underestimates because previous researchers used perceptual speed and psychomotor tests that are too brief to obtain adequate reliability (for a discussion of related issues, see Ackerman \& Cianciolo, 1999). 
Nonetheless, under explicit instructional emphasis towards accuracy, speed, or an equal emphasis on both accuracy and speed, a remarkably consistent set of results was found, which complicates the above conclusion about the relationship between test performance and $g$. Under accuracy-emphasis instructions, correlations between test item latency and $g$ were diminished, in comparison to both baseline measures and speed-emphasis instructional conditions. In contrast, error rate measures were most highly related to $g$ under accuracy-emphasis conditions, again both in comparison to the baseline assessments, and in comparison to the speed-emphasis conditions.

Raw correlations between the error rates and $g$ were relatively modest (except for the Mirror Tracing task), even under the accuracy-emphasis condition. Thus, even though the differences between the correlations under accuracy-emphasis and speed-emphasis conditions were significant, the amount of shared variance $\left(r^{2}\right)$ between the error measures and $g$ did not differ drastically between the two conditions. For the latency measures, however, the change in $r^{2}$ from the accuracy-emphasis to speed-emphasis was large enough to be noteworthy. For Scattered X's, the shared variance between the latency measure and $g$ increased modestly from $2.6 \%$ to $6.3 \%$, and for Summing to 10 , it went from $13.7 \%$ to $17.6 \%$. However, for the Eightitem SRT test, the shared variance went from $5.8 \%$ to $29.2 \%$, and for the Mirror Tracing test, it went from $6.8 \%$ to $33.6 \%$ from accuracy-emphasis to speed-emphasis conditions.

One might reasonably wonder how 'speeded' were the tests that composed the $g$ composite? The answer is that they were administered under standard timed-test conditions, but not under extreme time-stress. So, it is possible that some of the common variance between the perceptual speed and psychomotor test performance and $g$ is attributable to the demands of the 
reference tests, but this is a common feature of most (though not all) tests of intellectual abilities. It remains an open question whether the relatively high levels of common variance among these measures and $g$ would be found, if the $g$-reference tests were limited to untimed power tests.

Although Woodworth (1899) was perhaps the first to empirically identify individual differences in reactivity to conditions which demanded changes to speed and accuracy emphasis, there has been inadequate study of the issue in the subsequent decades. Nonetheless, there are some hints in the broader literature (e.g., Salthouse, 2000), that as adults age, there is a more substantial decline in speed of processing, in comparison to accuracy of responding, under traditional task instructions emphasizing both speed and accuracy. Whether this is represents an increase in rigidity associated with aging or a change in personal 'tempo' is not clear, nor is it known what specific physiological/neurological changes that might be responsible for such changes. In addition, there is evidence that lower-IQ individuals have more difficulty in regulating speed-accuracy tradeoffs, compared to normal-IQ individuals (e.g., see Brewer \& Smith, 1984), suggesting that there may be a more executive processing limitation associated with flexibility in strategic reactions to differential speed and accuracy demands. The current study does not allow for a more precise identification of the mechanisms underlying rigidity of personal tempo, but it does suggest that this is a promising avenue for future investigations of broader issues about the functional determinants of such individual differences.

\section{Conclusions}

\subsection{Scoring Tests vs. Instructional Manipulations}

Although we agree with Lohman (1989a) that it is not generally possible to determine, under normal testing conditions, where different individuals fall on the speed-accuracy tradeoff 
function, the current study is in disagreement about the notion that instructions for different emphasis on speed or accuracy are ineffective, at least for the kinds of perceptual speed and psychomotor tests examined in the current study. Thus, it appears possible that administering such tests under multiple instructional conditions may provide useful insights into individual differences in performance, from a more nuanced perspective of both accuracy and speed. The current study results show that such manipulations resulted in differential loadings on a $g$ composite, and as a consequence, one could reasonably expect changes to criterion-related validity indices as well. A more precise mapping of the speed-accuracy curve for each individual obviously would be more desirable than a sampling of three or more instructional conditions. However, the requirements for test design modifications to accomplish this task probably fall outside of the capabilities of many investigations, and appear to be limited to tests in which item exposure durations or response deadlines can be effectively imposed on the examinee.

\subsection{Test Situations and Underlying Constructs}

The current study illustrates that the construct validity of a test may be significantly affected by instructional conditions that are rarely considered to be important determinants of test performance. One might speculate that the kinds of instructions that are common to conventional speeded tests (e.g., "your score will be the number of items answered correctly minus a fraction for the items marked incorrectly) provides very limited information indeed to the examinee. Unless an examinee has a good understanding of his/her own personal speedaccuracy tradeoff function for the test content, and a better understanding of the scoring formula, it is possible that examinees may differ substantially in their relative emphasis for speed or 
accuracy. It is doubtful that variance attributable to such differences is appropriately relegated to error variance, because it may be systematic. It also may inflate the correlations of the target construct with other ability constructs, such as speediness (e.g., see Horn, 1965), that are not under consideration by the test developer (or even desirable from a test developer's perspective).

Consequences of imposed significant speed constraints on the examinee may have an impact on theory development and theory testing with respect to general intelligence too. In a meta-analysis of working memory and intelligence associations, Ackerman et al. $(2002,2005)$ found that where working memory tests were administered and correlated with performance on Raven's Progressive Matrices test, nearly all of the existing studies administered Raven's test in non-standard, highly-speeded conditions (that is, the Raven was designed as a power test, with relatively liberal time constraints). Inducing an emphasis on speed, as indicated in the current study, especially when the other (working memory) tests are also administered under significant speed-stress, may result in greater shared variance that is not, in and of itself, attributable to the intended underlying constructs. Rather, the observed relationship will be due to to some unknown combination of general ability construct variance and differential speed-accuracy tradeoff-related construct variance.

\subsection{Revisiting Speed vs. Level in the Context of Intelligence.}

With the exception of individual one-on-one intelligence assessments, such as the traditional Stanford-Binet and Wechsler-type IQ tests, group tests of abilities and computerbased testing have largely discarded the power test format, in favor of timed tests, which differ widely in terms of speed-stress. Because most highly speeded tests use items of homogeneous difficulty levels, they are not suited to adaptive testing (e.g., see Mead \& Drasgow, 1993). 
However, most computerized adaptive tests with heterogeneous item difficulties also have imposed time limits. Ultimately, the consideration of speed-accuracy tradeoff functions, both imposed (e.g., by instruction) and self-generated (through an individual's preferential strategy or through an individual's understanding of the costs and benefits associated with the penalties for incorrect answers), comes full circle to E. L. Thorndike and colleagues' (1926) assertion, which is in essence a ceteris paribus argument- that is, if examinees produce the same number of correct answers on a test, those that produce the answers faster are considered to be more intelligent. However, as noted extensively by Lohman and others, because examinees respond in such a way that they ordinarily are at different positions on their own speed-accuracy tradeoff curve, the ceteris paribus condition rarely occurs naturally during testing. Thus, speed and level of an individual's intellectual ability may be inextricably intertwined in many testing situations. We believe that the current study illustrates that there may be much to be learned about the nature of intellectual abilities, by focusing efforts on attempting to separate these two important components of test performance from a construct validity perspective (e.g., when investigating the relationship between speeded measures on one hand and general intelligence on the other hand), and also by implication, for criterion-related validity purposes. 


\section{References}

Ackerman, P. L. (1988). Determinants of individual differences during skill acquisition:

Cognitive abilities and information processing. Journal of Experimental Psychology: General, 117, 288-318.

Ackerman, P. L., \& Beier, M. E. (2007). Further explorations of perceptual speed abilities, in the context of assessment methods, cognitive abilities and individual differences during skill acquisition. Journal of Experimental Psychology: Applied, 13, 249-272.

Ackerman, P. L., Beier, M. E., \& Boyle, M. O. (2002). Individual differences in working memory within a nomological network of cognitive and perceptual speed abilities. Journal of Experimental Psychology: General, 131, 567-589.

Ackerman, P. L., Beier, M. E., \& Boyle, M. O. (2005). Working memory and intelligence: The same or different constructs? Psychological Bulletin, 131, 30-60.

Ackerman, P. L., Bowen, K. R., Beier, M. B., \& Kanfer, R. (2001). Determinants of individual differences and gender differences in knowledge. Journal of Educational Psychology, 93, 797-825.

Ackerman, P. L., \& Cianciolo, A. T. (2000). Cognitive, perceptual speed, and psychomotor determinants of individual differences during skill acquisition. Journal of Experimental Psychology: Applied, 6, 259-290.

Ackerman, P. L., \& Kanfer, R. (1993). Integrating laboratory and field study for improving selection: Development of a battery for predicting air traffic controller success. Journal of Applied Psychology, 78, 413-432.

Alderton, D. L., Wolfe, J. H., \& Larson, G. E. (1997). The ECAT battery. Military Psychology, 
9, 5-37.

Allison, R. B. (1960). Learning parameters and human abilities. Office of Naval Research Technical Report. Princeton, NJ: Educational Testing Service.

Beck, L. F. (1933). The role of speed in intelligence. Psychological Bulletin, 30 (2), 169-178.

Brewer, N., \& Smith, G. A. (1984). How normal and retarded individuals monitor and regulate speed and accuracy of responding in serial choice tasks. Journal of Experimental Psychology: General, 113, 71-93.

Cohen, J. (1990). Things I have learned (so far). American Psychologist, 45, 1304-1312.

Cureton, E. E., \& Cureton, L. W. (1955). The Multi-Aptitude Test. NY: Psychological Corporation.

Diamond, J., \& Evans, E. (1973). The correction for guessing. Review of Educational Research, 43 (2), 181-191.

Ekstrom, R. B., French, J. W., Harman, H. H., \& Dermen, D. (1976). Kit of factor-referenced cognitive tests. Princeton, NJ: Educational Testing Service.

Fleishman, E. A. (1953). Testing for psychomotor abilities by means of apparatus tests. Psychological Bulletin, 50, 241-263.

Horn, J. L. (1965). Fluid and crystallized intelligence: A factor analytic study of the structure among primary mental abilities. Unpublished doctoral dissertation: University of Illinois, Urbana, IL.

Jackson, D. N. (1984). Multidimensional Aptitude Battery. London, Ontario, Canada: Sigma Assessment Systems.

Jensen, A. R. (1998). The g factor: The science of mental ability. Westport, CT: Praeger. 
Jensen, A. R. (2006). Clocking the mind: Mental chronometry and individual differences. Amsterdam: Elsevier.

Lohman, D. F. (1986). The effect of speed-accuracy tradeoff on sex differences in mental rotation. Perception \& Psychophysics, 39, 427-436.

Lohman, D. F. (1989a). Individual differences in errors and latencies on cognitive tasks. Learning and Individual Differences, 1 (2) 179-202.

Lohman, D. F. (1989b). Estimating individual differences in information processing using speed-accuracy models. In R. Kanfer, P. L. Ackerman, \& R. Cudeck (Eds.) Abilities, motivation, and methodology: The Minnesota symposium on learning and individual differences (pp. 119-155). Hillsdale, NJ: Lawrence Erlbaum Associates.

Lord, F. M. (1975). Formula scoring and number-right scoring. Journal of Educational Measurement, 12 (1), 7-11.

Mead, A. D., \& Drasgow, F. (1993). Equivalence of computerized and paper-and-pencil cognitive ability tests: A meta-analysis. Psychological Bulletin, 114, 449-458.

Melton, A. W. (Ed.). Army Air Forces Aviation Psychology Program Research Reports: Apparatus Tests. Report No. 4. Washington, DC: U.S. Government Printing Office.

Quereshi, M. Y. (1960). Mental test performance as a function of payoff conditions, item difficulty, and degree of speeding. Journal of Applied Psychology, 44, 65-77.

Salthouse, T. A. (2000). Aging and measures of processing speed. Biological Psychology, 54, $35-54$.

Steiger, J. H. (1980). Tests for comparing elements of a correlation matrix. Psychological Bulletin, 87, 245-251. 
Sternberg, R. J. (1986). Haste makes waste versus a stitch in time? A reply to Vernon, Nador, and Kantor. Intelligence, 10 (3), 265-270.

Thorndike, E. L., Bregman, E. O., Cobb, M. V., \& Woodyard, E. (1926). The measurement of intelligence. Teachers College Columbia University: Bureau of Publications.

Thorndike, R. L. (1986). The role of general ability in prediction. Journal of Vocational Behavior, 29, 332-339.

Thurstone, L. L. (1937). Ability, motivation, and speed. Psychometrika, 2 (4), 249-254.

Thurstone, L. L., \& Thurstone, T. G. (1941). Factorial studies of intelligence. Psychometric Monograph, 2.

Tinker, M. A. (1931). The significance of speed in test response. Psychological Review, 38 (5), $450-454$.

Vernon, P. A., Nador, S., Kantor, L. (1985). Reaction times and speed-of-processing: Their relationship to timed and untimed measures of intelligence. Intelligence, 9 (4), 357-374.

Wickelgren, W. A. (1977). Speed-accuracy tradeoff and information processing dynamics. Acta Psychologica, 41, 67-85.

Woodworth, R. S. (1899). The accuracy of voluntary movement. Psychological Review Monograph Supplement, 3 (3) Whole No. 13. 
Table 1

Means, Standard Deviations, Internal Consistency Reliabilities, Correlations, and Factor Loadings for Reference Ability Tests

\begin{tabular}{lccccccccc}
\hline \multicolumn{1}{c}{ Variable } & $M$ & $S D$ & $r_{x x}$ & 1 & 2 & 3 & 4 & 5 & $\mathrm{I}$ \\
\hline 1. Vocabulary & 20.90 & 7.86 & $.90^{\mathrm{a}}$ & & & & & & .33 \\
2. Similarities & 26.13 & 4.81 & $.85^{\mathrm{b}}$ & $.57^{* *}$ & & & & & .50 \\
3. Math Approximation & 18.11 & 8.07 & $.90^{\mathrm{a}}$ & .15 & $.28^{* *}$ & & & & .81 \\
4. Arithmetic & 8.55 & 4.45 & $.90^{\mathrm{a}}$ & .18 & $.30^{* *}$ & $.78^{* *}$ & & & .72 \\
5. Paper Folding & 14.54 & 5.72 & $.85^{\mathrm{a}}$ & $.24^{*}$ & $.36^{* *}$ & $.63^{* *}$ & $.52^{* *}$ & & .78 \\
6. Spatial Analogy & 17.67 & 6.28 & $.90^{\mathrm{b}}$ & $.27^{* *}$ & $.44^{* *}$ & $.65^{* *}$ & $.51^{* *}$ & $.72^{* *}$ & .81 \\
\hline
\end{tabular}

Note. ${ }^{\text {a }}$ Reliability based on Part 1, Part 2 correlations and corrected with Spearman-Brown Prophecy formula; ${ }^{\mathrm{b}}$ Coefficient alpha; ${ }^{*} p<.05 ; * * p .01$. 
Table 2

Descriptive Statistics for Criterion Tests, and Repeated-measures ANOVA for Speed-accuracy Condition Means

Latency/Completion Time ${ }^{\mathrm{a}}$ (seconds)

\begin{tabular}{|c|c|c|c|c|c|c|c|c|c|c|}
\hline \multirow[b]{2}{*}{ Task } & \multicolumn{2}{|c|}{ Baseline } & \multicolumn{2}{|c|}{ Accuracy } & \multicolumn{2}{|c|}{ Balanced } & \multicolumn{2}{|c|}{ Speed } & \multirow[b]{2}{*}{$\mathrm{F}$} & \multirow[b]{2}{*}{$\eta_{p}^{2}$} \\
\hline & $M$ & $S D$ & $M$ & $S D$ & $M$ & $S D$ & $M$ & $S D$ & & \\
\hline Scattered X's & 5.38 & 1.47 & 4.70 & 1.89 & 3.91 & 1.11 & 3.33 & 1.03 & $49.08 * *$ & .34 \\
\hline Summing to 10 & 1.83 & 0.56 & 1.48 & 0.34 & 1.43 & 0.38 & 1.37 & 0.33 & $8.85 * *$ & .09 \\
\hline 8-item SRT & 3.69 & 0.48 & 3.78 & 0.50 & 3.53 & 0.43 & 3.41 & 0.44 & $59.19 * *$ & .38 \\
\hline Mirror Tracing & 31.72 & 12.67 & 21.99 & 6.41 & 19.60 & 5.08 & 17.71 & 5.12 & $33.25 * *$ & .26 \\
\hline
\end{tabular}

Errors $^{\mathrm{b}}$

\begin{tabular}{|c|c|c|c|c|c|c|c|c|c|c|}
\hline \multirow[b]{2}{*}{ Task } & \multicolumn{2}{|c|}{ Baseline } & \multicolumn{2}{|c|}{ Accuracy } & \multicolumn{2}{|c|}{ Balanced } & \multicolumn{2}{|c|}{ Speed } & \multirow[b]{2}{*}{$\mathrm{F}$} & \multirow[b]{2}{*}{$\eta_{\mathrm{p}}^{2}$} \\
\hline & $M$ & $S D$ & $M$ & $S D$ & $M$ & $S D$ & $M$ & $S D$ & & \\
\hline Scattered X's & 0.22 & 0.58 & 0.12 & 0.25 & 0.35 & 0.99 & 4.64 & 6.96 & $39.69 * *$ & .29 \\
\hline Summing to 10 & 0.65 & 0.73 & 0.35 & 0.44 & 0.83 & 1.06 & 5.59 & 12.22 & $16.30 * *$ & .15 \\
\hline 8-item SRT & 2.57 & 1.50 & 1.82 & 1.31 & 2.98 & 1.82 & 7.18 & 4.11 & $140.73 * *$ & .59 \\
\hline Mirror Tracing & 5.85 & 2.66 & 2.84 & 1.93 & 4.34 & 2.14 & 7.12 & 2.42 & $195.90 * *$ & .68 \\
\hline
\end{tabular}

Note: SRT = Serial Reaction Time; ${ }^{2}$ Latency/Completion Time: latency = time in seconds to find one item in Scattered X's and Summing to 10; completion time $=$ time in seconds to complete one trial. ${ }^{b}$ Errors represents the mean number of errors per 5-minute test part (Scattered X's and Summing to 10), or mean number of errors per trial (Mirror Tracing) or block of trials (8-item SRT). $* * p<.01$. Reliability estimates (based on Spearman-Brown Prophecy Formula for the baseline conditions of each test (latency/completion time and errors, respectively)) are as follows: Scattered X's (.95, .70), Summing to 10 (.96, .96), 8-item SRT (.98, $81)$ and Mirror Tracing $(.92, .86)$. 
Table 3

Correlations Between g Composite and Latency, Error Measures for Criterion Tests.

$$
\text { Latency/Completion Time }
$$

\begin{tabular}{|c|c|c|c|c|c|}
\hline Task & Baseline & Accuracy & Balanced & Speed & Williams' $\mathrm{T}^{\mathrm{a}}$ \\
\hline Scattered X's & $-.32 * *$ & -.16 & $-.23 *$ & $-.25 *$ & -0.88 \\
\hline Summing to 10 & $-.51 * *$ & $-.37 * *$ & $-.44 * *$ & $-.42 * *$ & -0.70 \\
\hline 8-item SRT & $-.48 * *$ & $-.24 *$ & $-.55 * *$ & $-.54 * *$ & $-4.11 * *$ \\
\hline Mirror Tracing & $-.62 * *$ & $-.26 *$ & $-.49 * *$ & $-.58 * *$ & $-3.34 * *$ \\
\hline
\end{tabular}

\begin{tabular}{lllllc}
\multicolumn{1}{c}{ Task } & Baseline & Accuracy & Balanced & Speed & Williams' T $^{\mathrm{a}}$ \\
\hline Scattered X's & $-.28^{* *}$ & $-.31^{* *}$ & -.08 & .03 & $2.78^{* *}$ \\
Summing to 10 & -.05 & $-.27^{* *}$ & -.11 & .09 & $2.86^{* *}$ \\
8-item SRT & -.02 & $-.34^{* *}$ & .00 & .13 & $4.18^{* *}$ \\
Mirror Tracing & $-.50^{* *}$ & $-.50^{* *}$ & $-.37 * *$ & $-.22^{*}$ & $2.95^{* *}$ \\
\hline
\end{tabular}

Note. $d f$ for Williams' T test $=92-95$ (due to missing data). ${ }^{\mathrm{a}}$ Williams' $\mathrm{T}$ tests comparing correlations under accuracy instructions with correlations under speed instructions. ${ }^{*} p<.05 ;{ }^{*} p<.01$ 
Figure 1. Static screen captures of the four perceptual speed and psychomotor tests. Clockwise from the upper left: Scattered X's test, Summing to 10 test, Eight-item Serial Reaction Time test, and Mirror Tracing test.

Figure 2. Correlations between perceptual speed/psychomotor tests and $g$ composite for baseline and speed-accuracy emphasis instructional conditions. Correlations with latency/speed variables in solid lines and circles. Correlations with error variables in dashed lines and squares. 


\section{Scattered X's}

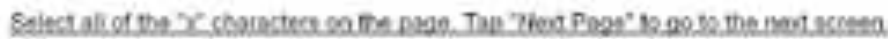

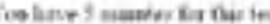

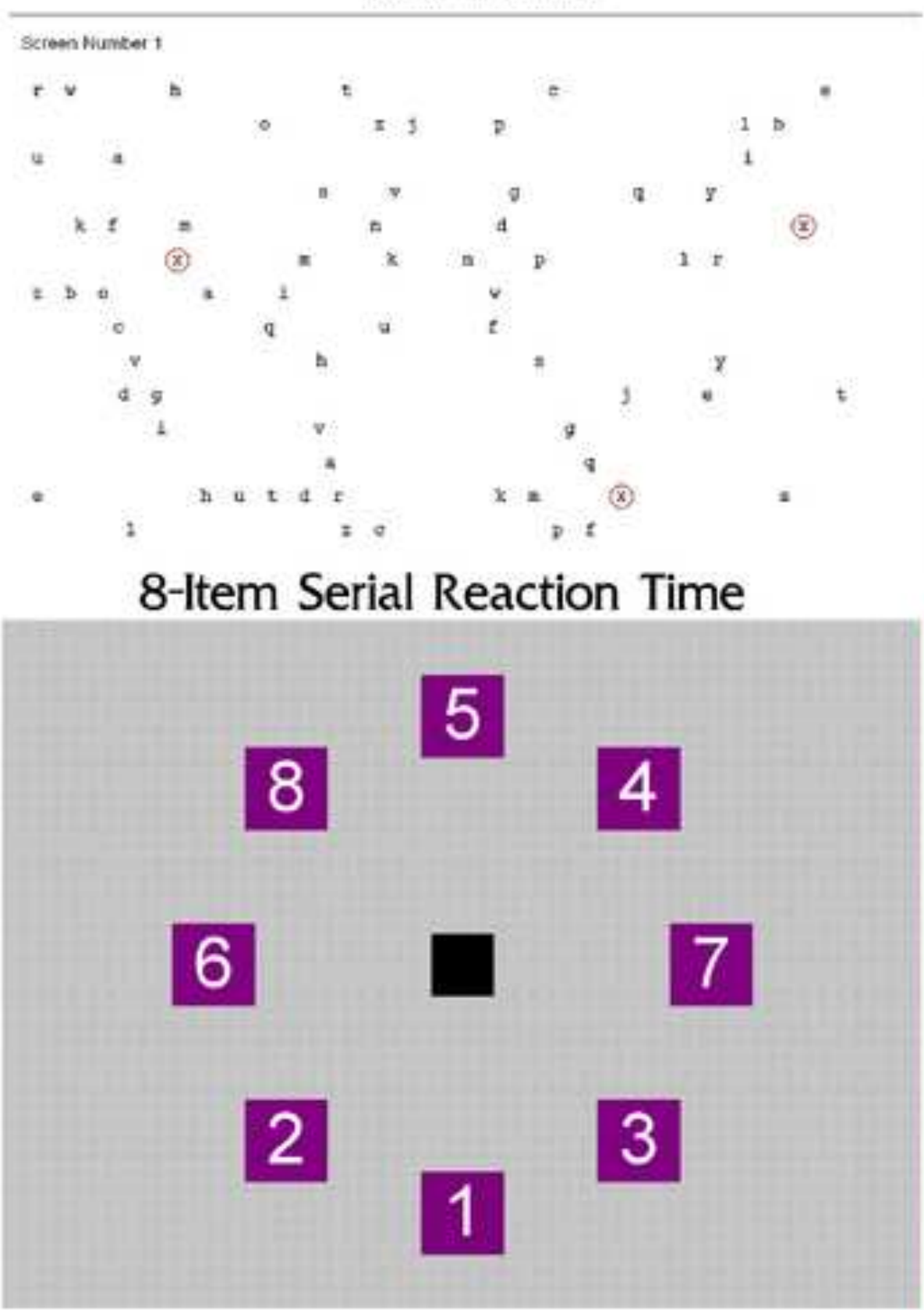

Summing to 10

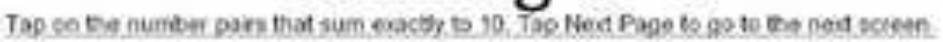

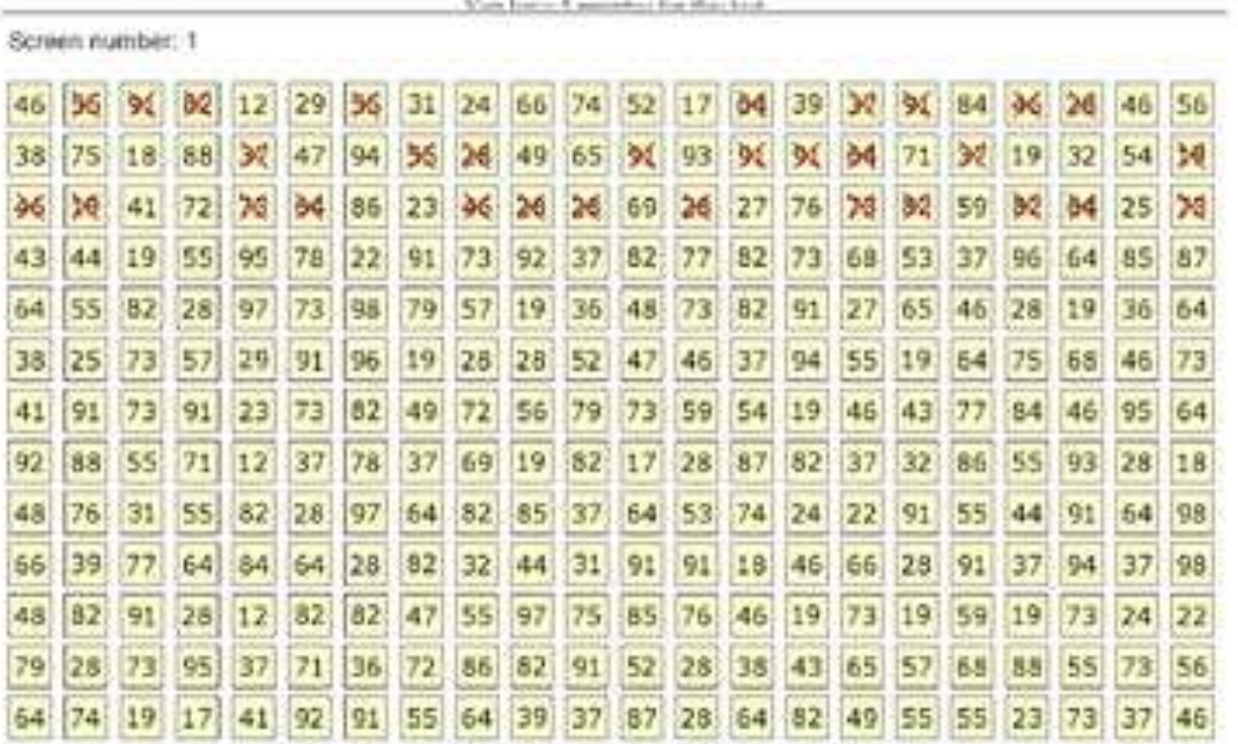

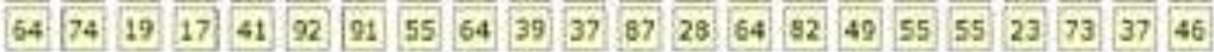

\section{Mirrror Tracing}

\section{Mirror Tracing Task}

Put your touch-pen here

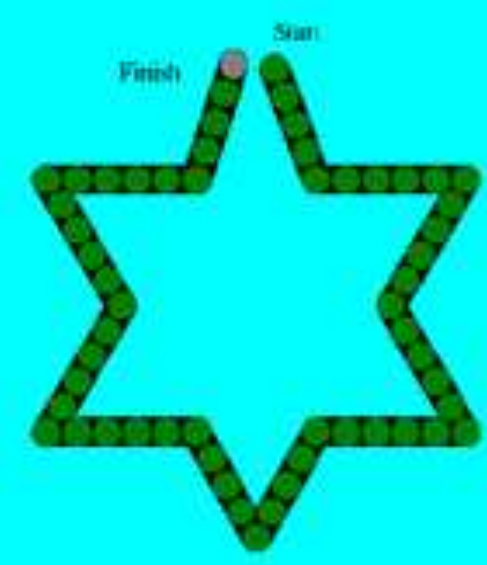



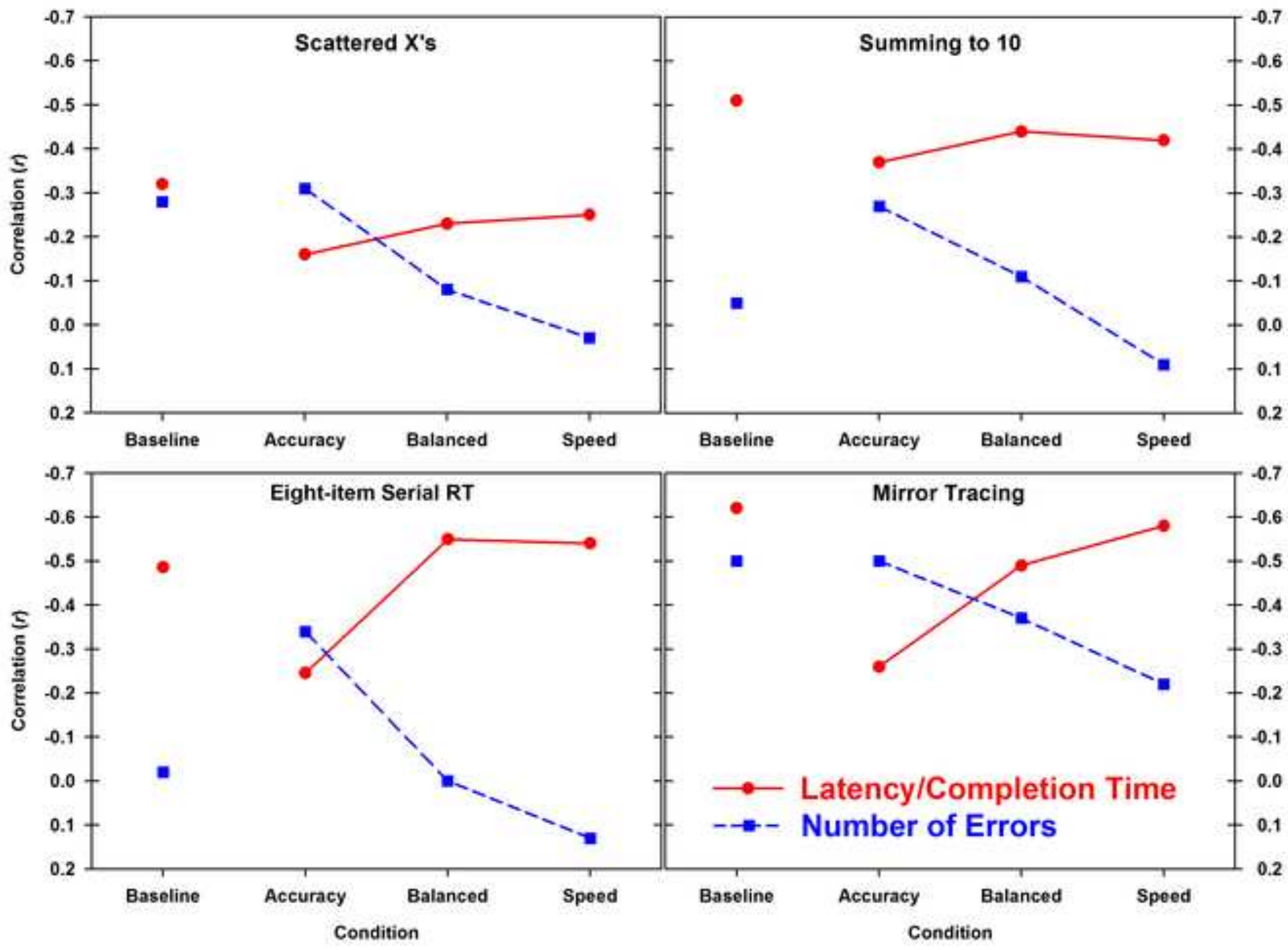